\title{
Addendum to "Sufficient conditions for three-particle entanglement and their tests in recent experiments"
}

\author{
Géza Tóth, ${ }^{1, *}$ Otfried Gühne, ${ }^{2, \dagger}$ Michael Seevinck, ${ }^{3, \$}$ and Jos Uffink ${ }^{3, \S}$ \\ ${ }^{1}$ Max-Planck-Institut für Quantenoptik, Hans-Kopfermann-Straße 1, D-85748 Garching, Germany \\ ${ }^{2}$ Institut für Quantenoptik und Quanteninformation, Österreichische Akademie der Wissenschaften, A-6020 Innsbruck, Austria \\ ${ }^{3}$ Institute of History and Foundations of Science, Utrecht University, P.O. Box 80.000, 3508 TA Utrecht, The Netherlands
}

(Received 12 April 2005; published 8 July 2005)

\begin{abstract}
A recent paper [M. Seevinck and J. Uffink, Phys. Rev. A 65, 012107 (2002)] presented a bound for the three-qubit Mermin inequality such that the violation of this bound indicates genuine three-qubit entanglement. We show that this bound can be improved for a specific choice of observables. In particular, if spin observables corresponding to orthogonal directions are measured at the qubits (e.g., $X$ and $Y$ spin coordinates), then the bound is the same as the bound for states with a local hidden variable model. As a consequence, it can straightforwardly be shown that in the experiment described by J.-W. Pan et al. [Nature 403, 515 (2000)], genuine three-qubit entanglement was detected.
\end{abstract}

DOI: $10.1103 /$ PhysRevA.72.014101

PACS number(s): 03.65.Ud

Before presenting our comments concerning Ref. [1], let us briefly recall the difference between genuine three-qubit entanglement, partial (biseparable) entanglement, and full separability. A pure state is called fully separable if it is of the product form $|\psi\rangle=|a\rangle|b\rangle|c\rangle$. It is called biseparable if a partition of the qubits into two groups can be found, for which the state is separable, while for genuine three-qubit entangled states this is not possible. For example, the state $(|000\rangle+|110\rangle) / \sqrt{2}=(|00\rangle+|11\rangle)|0\rangle / \sqrt{2}$ is biseparable since the third qubit is not entangled with the first two, while $|G H Z\rangle=(|000\rangle+|111\rangle) / \sqrt{2}$ is a state with genuine three-qubit entanglement [2].

Let us now consider the three-qubit Mermin inequality. Its Bell operator is [3]

$$
M_{3}:=X^{(1)} X^{(2)} X^{(3)}-Y^{(1)} Y^{(2)} X^{(3)}-X^{(1)} Y^{(2)} Y^{(3)}-Y^{(1)} X^{(2)} Y^{(3)},
$$

where $X$ and $Y$ are Pauli spin matrices. Note that these observables correspond to orthogonal measurement directions. For states allowing a local hidden variable model, it is known that the Mermin inequality $\left|\left\langle M_{3}\right\rangle\right| \leqslant 2$ has to hold [3]. Consequently, all fully separable states have to obey the same inequality, since they allow for a local hidden variable model.

Let us now show that for biseparable states the bound is also 2, thus $\left|\left\langle M_{3}\right\rangle\right|>2$ implies that the state carries genuine tripartite entanglement. For biseparable pure states of the form $\Psi=\Psi_{12} \otimes \Psi_{3}$ we have

$$
\begin{aligned}
\left\langle M_{3}\right\rangle= & \left\langle X^{(1)} X^{(2)}\right\rangle\left\langle X^{(3)}\right\rangle-\left\langle Y^{(1)} Y^{(2)}\right\rangle\left\langle X^{(3)}\right\rangle-\left\langle X^{(1)} Y^{(2)}\right\rangle\left\langle Y^{(3)}\right\rangle \\
& -\left\langle Y^{(1)} X^{(2)}\right\rangle\left\langle Y^{(3)}\right\rangle .
\end{aligned}
$$

Now $\left\langle M_{3}\right\rangle$ is given with six operator expectation values. We

\footnotetext{
*Electronic address: toth@alumni.nd.edu

†Electronic address: otfried.guehne@uibk.ac.at

†Electronic address: m.p.seevinck@ phys.uu.nl

${ }^{\S}$ Electronic address: J.B.M.Uffink@ @hys.uu.nl
}

are looking for its maximum, with the constraint that these expectation values have physically accessible values. That is, for example, the values for $\left\langle X^{(3)}\right\rangle$ and $\left\langle Y^{(3)}\right\rangle$ obey $\left\langle X^{(3)}\right\rangle^{2}$ $+\left\langle Y^{(3)}\right\rangle^{2} \leqslant 1$. Let us now define two vectors with the expectation values as

$$
\begin{gathered}
\vec{v}_{1}:=\left(\left\langle X^{(1)} X^{(2)}-Y^{(1)} Y^{(2)}\right\rangle,-\left\langle X^{(1)} Y^{(2)}+Y^{(1)} X^{(2)}\right\rangle\right), \\
\vec{v}_{2}:=\left(\left\langle X^{(3)}\right\rangle,\left\langle Y^{(3)}\right\rangle\right) .
\end{gathered}
$$

The relevant constraints for physical states are summarized in $\left|\vec{v}_{1}\right| \leqslant 2[8]$ and $\left|\vec{v}_{2}\right| \leqslant 1$. One easy way for getting an upper bound for $\left|\left\langle M_{3}\right\rangle\right|$ is using the Cauchy-Schwarz inequality

$$
\left|\left\langle M_{3}\right\rangle\right|=\left|\vec{v}_{1} \cdot \vec{v}_{2}\right| \leqslant\left|\vec{v}_{1}\right|\left|\vec{v}_{2}\right| \leqslant 2,
$$

where $\cdot$ denotes scalar product. Since $M_{3}$ is invariant under permuting qubits, this bound is clearly valid also for biseparable pure states with partitions (1) (23) and (13) (2). It is easy to see that the bound is also valid for the mixture of pure biseparable states [4].

In Ref. [1] it was shown that for biseparable quantum states

$$
\left|\left\langle M_{3}\right\rangle\right| \leqslant 2 \sqrt{2}
$$

has to hold, and that violation of this bound implies genuine tripartite entanglement. This bound was found by considering the following expression:

$$
\Sigma:=\left|E(a, b, c)-E\left(a^{\prime}, b^{\prime}, c\right)-E\left(a, b^{\prime}, c^{\prime}\right)-E\left(a^{\prime}, b, c^{\prime}\right)\right|,
$$

where $a, b, c, a^{\prime}, b^{\prime}, c^{\prime}$ are arbitrary dichotomic observables on each qubit and $E(a, b, c)$ denotes the expectation value of the corresponding product $a \cdot b \cdot c$, if $a$ is measured at the first qubit, $b$ is at the second, etc. The upper bound of $\Sigma$ for fully separable states is known to be $\Sigma \leqslant 2$.

References $[1,5,6]$ show that for biseparable quantum states we have $\Sigma \leqslant 2 \sqrt{2}$. Whether this bound is sharp can be checked by looking for states for which $\Sigma=2 \sqrt{2}$. Such a state is presented in Ref. [1]: For this example $c=c^{\prime}$. Thus this 
bound is sharp if we allow arbitrary observables to be measured at the qubits. When the observables are fixed to be $X$ and $Y$, the bound can be improved, as we have seen above. The bound in our new inequality $\left|\left\langle M_{3}\right\rangle\right| \leqslant 2$ is sharp since for the biseparable state $(|00\rangle+|11\rangle)(|0\rangle+|1\rangle) / 2$ we have $\left|\left\langle M_{3}\right\rangle\right|=2$.

In Ref. [1] several experiments were discussed and it was investigated, whether three-qubit entanglement was present in these experiments. The experiment described in Ref. [7] aimed to create a GHZ state with three photons. Here the horizontal and vertical polarization of the photons encoded the one-qubit information. After the state was created, the polarization of the photons was measured. In particular, for the expectation value of $M_{3}$ they obtained 2.83 \pm 0.09 . Based on our present discussion, we can say, based merely on the value measured for $\left\langle M_{3}\right\rangle$, that three-qubit entanglement has been detected in these experiments.

In this Addendum, we have shown that the general bound obtained in Ref. [1] for biseparable states can be improved for the specific choice of orthogonal observables as in the three-qubit Mermin inequality. We determined the sharp bound for this case. We showed that the three-qubit Mermin inequality can be used to detect genuine three-qubit entanglement with the same bound which is used to detect the violation of local realism.

This sheds new light on old experimental data in Ref. [7] and shows that genuine three-qubit entanglement has already been realized experimentally [9]. Our discussion also has a message for using the Mermin inequality to detect genuine multiparticle entanglement in future experiments.

We would like to thank M. Aspelmeyer, J. I. Cirac, J.-W. Pan, H. Weinfurter, A. Zeilinger, and M. Zukowski for useful discussions. We also acknowledge the support of the EU project RESQ and QUPRODIS and the Kompetenznetzwerk Quanteninformationsverarbeitung der Bayerischen Staatsregierung. G.T. thanks the Marie Curie Individual Fellowship of the European Union (Grant No. MEIF-CT-2003-500183).
[1] M. Seevinck and J. Uffink, Phys. Rev. A 65, 012107 (2002).

[2] Consequently, a mixed state is called biseparable (fully separable) if it is the mixture of pure biseparable (fully separable) states.

[3] N. D. Mermin, Phys. Rev. Lett. 65, 1838 (1990).

[4] For a different proof see Géza Tóth and Otfried Gühne, Phys. Rev. Lett. 94, 060501 (2005); Géza Tóth and Otfried Gühne, quant-ph/0501020.

[5] N. Gisin and H. Bechmann-Pasquinucci, Phys. Lett. A 246, 1 (1998).

[6] D. Collins, N. Gisin, S. Popescu, D. Roberts, and V. Scarani, Phys. Rev. Lett. 88, 170405 (2002).

[7] J.-W. Pan, D. Bouwmeester, M. Daniell, H. Weinfurter, and A. Zeilinger, Nature (London) 403, 515 (2000); see also p. 209 of D. Bouwmeester, A. Ekert and Z. Zeilinger, The Physics of Quantum Information (Springer, Berlin, 2000).

[8] This can be proved as follows. Let us take $\rho$ to be a $4 \times 4$ matrix in the usual $Z^{(k)}$ basis, with matrix elements $\rho_{k l}$ with $k, l=1,2,3,4$. Then $\left|v_{1}\right|^{2}=\left(\left\langle X^{(1)} X^{(2)}-Y^{(1)} Y^{(2)}\right\rangle_{\rho}\right)^{2}+\left(\left\langle X^{(1)} Y^{(2)}\right.\right.$ $\left.\left.+Y^{(1)} X^{(2)}\right\rangle_{\rho}\right)^{2}=16 \rho_{14} \rho_{41} \leqslant 4$. The last inequality is valid since $\left|\rho_{14}\right|=\left|\rho_{41}\right| \leqslant 1 / 2$ is required for physically meaningful density matrices. See also Eq. (3) of J. Uffink, Phys. Rev. Lett. 88, 230406 (2002).

[9] We note that in a long calculation using the fidelity criterion, K. Nagata, M. Koashi, and N. Imoto, proved that the experiment contained three-particle entanglement but without using the Mermin inequality: K. Nagata, M. Koashi, and N. Imoto, Phys. Rev. A 65, 042314 (2002); as we have recently been informed in a calculation different from ours, K. Nagata, M. Koashi, and N. Imoto, also obtained the here presented bound for the Mermin inequality in an unpublished work to be found at http://www.qci.jst.go.jp/eqis02/program/abstract/ poster11.pdf. The bound, while not explicitly stated, can straightforwardly be obtained from K. Nagata, M. Koashi, and N. Imoto, Phys. Rev. Lett. 89, 260401 (2002). 